\title{
Sensitive Liquid Chromatography/Tandem Mass Spectrometry Method for the Simultaneous Determination of Risperidone, Olanzapine, Quetiapine, Clozapine, Ziprasidone, Perospirone, Aripiprazole and Blonanserin in Human Serum
}

\author{
Keiko Tonooka1 ${ }^{*}$, Lynn Yoshida1, Koji Tomobe, Yoko Kunisue², Masaru Terada1, \\ Tatsuo Shinozuka ${ }^{1}$
}

${ }^{1}$ Department of Pathophysiology, Yokohama University of Pharmacy, Kanagawa, Japan ${ }^{2}$ Foundation for Promotion of Material Science and Technology of Japan, Tokyo, Japan Email: *k.tonooka@hamayaku.ac.jp

How to cite this paper: Tonooka, K., Yoshida, L., Tomobe, K., Kunisue, Y., Terada, M. and Shinozuka, T. (2018) Sensitive Liquid Chromatography/Tandem Mass Spectrometry Method for the Simultaneous Determination of Risperidone, Olanzapine, Quetiapine, Clozapine, Ziprasidone, Perospirone, Aripiprazole and Blonanserin in Human Serum. American Journal of Analytical Chemistry, 9, 88-97.

https://doi.org/10.4236/ajac.2018.92008

Received: December 27, 2017

Accepted: February 24, 2018

Published: February 27, 2018

Copyright (c) 2018 by authors and Scientific Research Publishing Inc. This work is licensed under the Creative Commons Attribution-NonCommercial International License (CC BY-NC 4.0). http://creativecommons.org/licenses/by-nc/4.0/ (c) (i) \& Open Access

\begin{abstract}
The sensitive method for the simultaneous determination of eight atypical antipsychotic drugs (risperidone, olanzapine, quetiapine, clozapine, ziprasidone, perospirone, aripiprazole and blonanserin) in human serum has been developed based on a high-performance liquid chromatography-tandem mass spectrometry (LC/MS/MS) with electrospray ionization (ESI). The chromatographic separation was performed on a Mightysil-RP-18 MS column $(2.0 \mathrm{~mm} \times$ $150 \mathrm{~mm}$, particle size $3 \mu \mathrm{m}$ ). The mobile phase consisted of $10 \mathrm{mM}$ formic ammonium buffer ( $\mathrm{pH}$ 6.0) and acetonitrile and was delivered at a flow rate of $0.20 \mathrm{~mL} / \mathrm{min}$. The triple quadrupole mass spectrometer was operated in positive ion mode, and multiple reaction monitoring was used for drug quantification. Solid-phase extraction of the eight antipsychotic drugs added to the human serum was performed with an Oasis HLB extraction cartridges column. The method was linear for the investigated drugs over the concentration range of $20-100 \mathrm{ng} / \mathrm{mL}$. The recoveries of these drugs were in the range of $81.3 \%-140 \%$. The intraday and inter day standard deviation (SD) values for all analytes were $<0.10$. Therefore, the selectivity, accuracy and precision of this method are sufficient for clinical and forensic studies. This simultaneous screening method will be also applicable to analyze other atypical antipsychotic drugs.
\end{abstract}




\section{Keywords}

LC/MS/MS, Atypical Antipsychotic Drugs, Analysis, Solid Phase Extraction

\section{Introduction}

The atypical antipsychotic drugs are one of the groups of antipsychotics used to treat psychiatric conditions. They are prescribed much more frequently now and became a first-line agent for schizophrenia as they show less side-effect than the primarily used typical antipsychotic drugs [1]. These drugs are metabolized by the liver extensively and excreted into the urine in animals and humans.

In forensic toxicology, atypical antipsychotics are of considerable interest because of their abusive potential such as their involvement in intoxications and suicides. In recent years, because several new drugs dosed at low levels have also become available, there has been an increasing need of analytical methods in order to analyze biological samples.

Fatal poisoning due to these drugs is often reported. Because of their combination misuse or excessive dosage, various intoxication cases have been reported to date [2] [3] [4] [5]. Therefore, it is important to develop a simple and sensitive way to analyze these drugs.

Several methods for the determination of atypical antipsychotic drugs using high-performance liquid chromatography (HPLC) [6], LC/MS and LC/MS/MS have already been reported [7] [8] [9] [10] [11]. However, the simultaneous determination of eight of them (risperidone, olanzapine, quetiapine, clozapine, ziprasidone, perospirone, aripiprazole and blonanserin) using LC/MS/MS has not been seen.

Here for the first time, we developed a selective and sensitive way to determine eight atypical antipsychotic drugs at the same time, and a simple method for solid-phase column extraction of the drugs from human serum using an Oasis HLB cartridge column.

\section{Experimental Procedures}

\subsection{Chemicals}

Eight atypical antipsychotic drugs were examined in this study. Drug standards for aripiprazole, blonanserin, clozapine, perospironehydrochloride dehydrate and ziprasidone hydrochloride were purchased from Waco Pure Chemical Industries (Osaka, Japan). Olanzapine, quetiapinehemifumarate and risperidone were purchased from Tokyo Chemical Industry Co., Ltd. (Tokyo, Japan). Clozapine- $d_{4}$ (internal standard: IS) was purchased from Sigma-Aldrich (Tokyo, Japan). Normal human serum was purchased from Millipore (Temecula, CA, USA). Methanol (HPLC grade), water (HPLC grade), acetonitrile (HPLC grade), acetic acid (analytical grade) and ammonium acetate (analytical grade) were purchased from Wako Pure Chemical Industries Ltd. (Osaka, Japan). 
Oasis HLB extraction cartridges were purchased from Waters (Milford, MA, USA). All other chemicals were commercially available and of reagent grade.

\subsection{Preparation of the Standard Solutions and Calibration Standards}

Individual stock solutions of the analyte compounds at a concentration of 1 $\mathrm{mg} / \mathrm{mL}$ were prepared in methanol (stable for at least three months when stored at $-20^{\circ} \mathrm{C}$ ). Serum standards were prepared with concentrations of $20,40,60,80$ and $100 \mathrm{ng} / \mathrm{mL}$ of each compound by diluting appropriate aliquots of the stock solution with drug-free serum (normal human serum). The calibration curves were obtained by simple linear regression analysis of each drug's concentration and corresponding peak area ratio. The regression equations for the eight atypical antipsychotic drugs extracted from human serum are based on the ratio of peak area of each drug to that of the IS.

\subsection{Apparatus and Chromatographic Conditions}

LC/MS/MS experiments were performed with an HPLC system, which consisted of Shimadzu LC-20AD pumps (Shimazu, Kyoto, Japan), a SIL-20ACautosampler (Columbia, MD, USA) and the 4000QTRAPmass spectrometer (Applied Biosystems, Foster City, CA, USA).

The chromatographic separation was performed on a Mightysil-RP-18 MS column $(2.0 \mathrm{~mm} \times 150 \mathrm{~mm}$, particle size $3 \mu \mathrm{m}$; Kanto Chemical Co., Inc., Tokyo, Japan) with a guard cartridge $(2.0 \mathrm{~mm})$. For the gradient elution, two solvents were used: (A) $10 \mathrm{mM}$ formic ammonium buffer, $\mathrm{pH} 6.0$ and (B) acetonitrile. The mobile phase composition was held initially at $60 \%$ (A) for $20 \mathrm{~min}$ and then changed during the linear gradient elution from $60 \%$ (A) to $30 \%$ (A) over 10 $\min$. The mobile phase was then returned to $60 \%$ (A) over 5 min for the next run. The flow rate was set to $0.20 \mathrm{~mL} / \mathrm{min}$ and $10 \mu \mathrm{L}$ of sample was injected for each analysis. The column and autosampler were maintained at 37 and $4^{\circ} \mathrm{C}$, respectively.

All experiments were conducted in the positive ion electrospray mode. The TurboIonSpray source was operated at $600^{\circ} \mathrm{C}$ with the capillary voltage set at $5500 \mathrm{~V}$. Nitrogen was used as the nebulizer gas, curtain gas (40 psi) and collision gas (4 psi). The collision energy was set between at 20 and $35 \mathrm{~V}$. The instrument was used in Multiple Reaction Monitoring (MRM) mode (Table 1).

\subsection{Extraction Procedure of the Drugs from Human Serum Using an Oasis ${ }^{\circledR}$ HLB Cartridge Column}

The human serum samples were stored at $-20^{\circ} \mathrm{C}$ until analysis. These samples were analyzed to determine possible endogenous interferences and were used as "blanks". An Oasis HLB cartridge column with a capacity of $1 \mathrm{~mL}$ was placed in an Agilent VacElut system (Agilent Technologies, CA, USA). Each column was activated by washing with $1 \mathrm{~mL}$ of methanol followed by $1 \mathrm{~mL}$ of water. 
Table 1. Retention time and tandem mass spectrometry parameters of atypical antipsychotic drugs.

\begin{tabular}{ccccc}
\hline Drugs & $\begin{array}{c}\text { Retention time } \\
(\mathrm{min})\end{array}$ & MW & Precursor ion $^{\mathrm{a}}$ & Product ion \\
\hline Risperidone & 2.66 & 410.49 & 411 & 191 \\
Olanzapine & 4.23 & 312.44 & 313 & $256,84,213,282$ \\
Quetiapine & 4.78 & 383.51 & 384 & $253,279,158$ \\
Clozapine & 5.59 & 326.82 & 328 & $271,84,297,229$, \\
Ziprasidone & 5.77 & 412.94 & 414 & $194,177,221$ \\
Perospirone & 6.60 & 426.57 & 427 & $177,208,167,251$ \\
Aripiprazole & 8.64 & 448.39 & 449 & $286,176,219$ \\
Blonanserin & 16.97 & 367.50 & 368 & $297,325,98$ \\
Clozapine- $d_{4}(\mathrm{IS})$ & 5.53 & 330.15 & 331 & 272 \\
\hline
\end{tabular}

${ }^{\mathrm{a} Q 1 .}$

Five microliters of phosphoric acid and $500 \mu \mathrm{L}$ of water were added to $1.0 \mathrm{~mL}$ of human serum containing $20-100 \mathrm{ng}$ of each of the eight atypical antipsychotic drugs and $100 \mathrm{ng}$ clozapine- $d_{4}$ (IS). The mixture was applied to the Oasis HLB cartridge column and allowed to pass through. The column was then washed with $1 \mathrm{~mL}$ of $2 \%$ ammonium hydroxide in a $10 \%$ methanol solution. The atypical antipsychotic drugs were eluted with $1 \mathrm{~mL}$ of a methanol: $2 \%$ acetic acid (70:30) solution. The eluent was dried under $\mathrm{N}_{2}$ gas at $40^{\circ} \mathrm{C}$. The residue was dissolved in $200 \mu \mathrm{L}$ of mobile phase, and an aliquot $(10 \mu \mathrm{L})$ was then analyzed by the chromatographic system.

\subsection{Linearity, Accuracy, Precision, and Recovery}

For the evaluation of the linearity of the standard calibration curve, the analyses of the eight atypical antipsychotic drugs in serum samples were performed on three separate days using freshly prepared samples and solutions. The calibration curves were prepared over a linear range of $20-100 \mathrm{ng} / \mathrm{mL}$ using five concentrations $(20,40,60,80$ and $100 \mathrm{ng} / \mathrm{mL})$.

The intraday assay precision and accuracy were obtained by analyzing six aliquots of the quality control samples in duplicate using a calibration curve constructed on the same day. The inter day assay precision and accuracy were evaluated by six replicate determinations for each concentration with solutions made on different days. The recoveries were calculated by comparing the chromatographic peak areas obtained from the extracts of the serum samples (containing the eight atypical antipsychotic drugs and the IS) with those obtained by extracting the drugs from water instead of serum.

\subsection{Stability}

The stability of the eight atypical antipsychotic drugs and the IS in serum was investigated at various concentrations. Freshly prepared samples were evaluated 
at room temperature, $4^{\circ} \mathrm{C}$ and $-20^{\circ} \mathrm{C}$ over 14 days. The freeze/thaw stability was determined after three freeze/thaw cycles. Stability tests were assessed by analyzing two replicates for each sample.

\subsection{Limits of Detection and Quantification (LOD and LOQ)}

The limit of detection (LOD) was determined from the injection volume required to produce a peak with a signal/noise ratio of at least 3:1. The limit of quantification (LOQ) is the lowest concentration on the standard curve which can be measured with acceptable accuracy (standard deviation, SD $<0.10$ ). The LOQ for the eight drugs in serum was determined by LC/MS/MS with ESI after solid-phase extraction (Oasis HLB).

\section{Results and Discussion}

\subsection{Selectivity and Chromatography}

Clear separation peaks of eight drugs by LC/MS/MS were seen on the chromatograms (Figure 1). The positive ion electrospray mode was selected, optimal collision energies were optimized for each drug (Figure 2). All precursor ion (Q1) of eight drugs showed the $[\mathrm{M}+\mathrm{H}]^{+}$. The $[\mathrm{M}+\mathrm{H}]^{+}$ion peak was detected clearly and product ion were obtained in the mass spectrum of each drug, with a sufficient detection sensitivity (Table 1, Figure 2).

\subsection{Linearity, Accuracy, Precision, and Recovery}

The calibration curve (the ratio of the peak area and the concentration of each drug) was linear over the concentration range of $20-100 \mathrm{ng} / \mathrm{mL}$. The coefficients of determination ( $r$ ) from the regression analyses of the drugs were between 0.9177 and 0.9986 (Table 2).

Nirogi R. V. et al. [11] developed a method for the determination of atypical antipsychotic drug in human plasma by LC/MS/MS with ESI. Following liquid-liquid extraction, the recovery was approximately $85.5 \%( \pm 1.9 \%)$ for olanzapine.

In the our LC/MS/MS with ESI method for simultaneous determination of eight atypical antipsychotic drugs, the SD values were always lower than 0.10 for the intraday, inter day and total assay reproducibility, showing the acceptable precision of this method. No significant interferences were also observed at the retention times of the analytes and IS, indicating its high selectivity. The recoveries of the drugs were ranged from $81.3 \%$ to $140 \%$ (Table 3 ) and the matrix effect was insignificant.

\subsection{Stability}

A stability study was conducted to determine the best storage temperature for the serum samples. The results indicated that the eight drugs and the IS were stable up to $12 \mathrm{~h}$ at room temperature. Furthermore, these drugs were stable up to three weeks when stored at $4^{\circ} \mathrm{C}$ and $-20^{\circ} \mathrm{C}$. Therefore, all extracted samples 

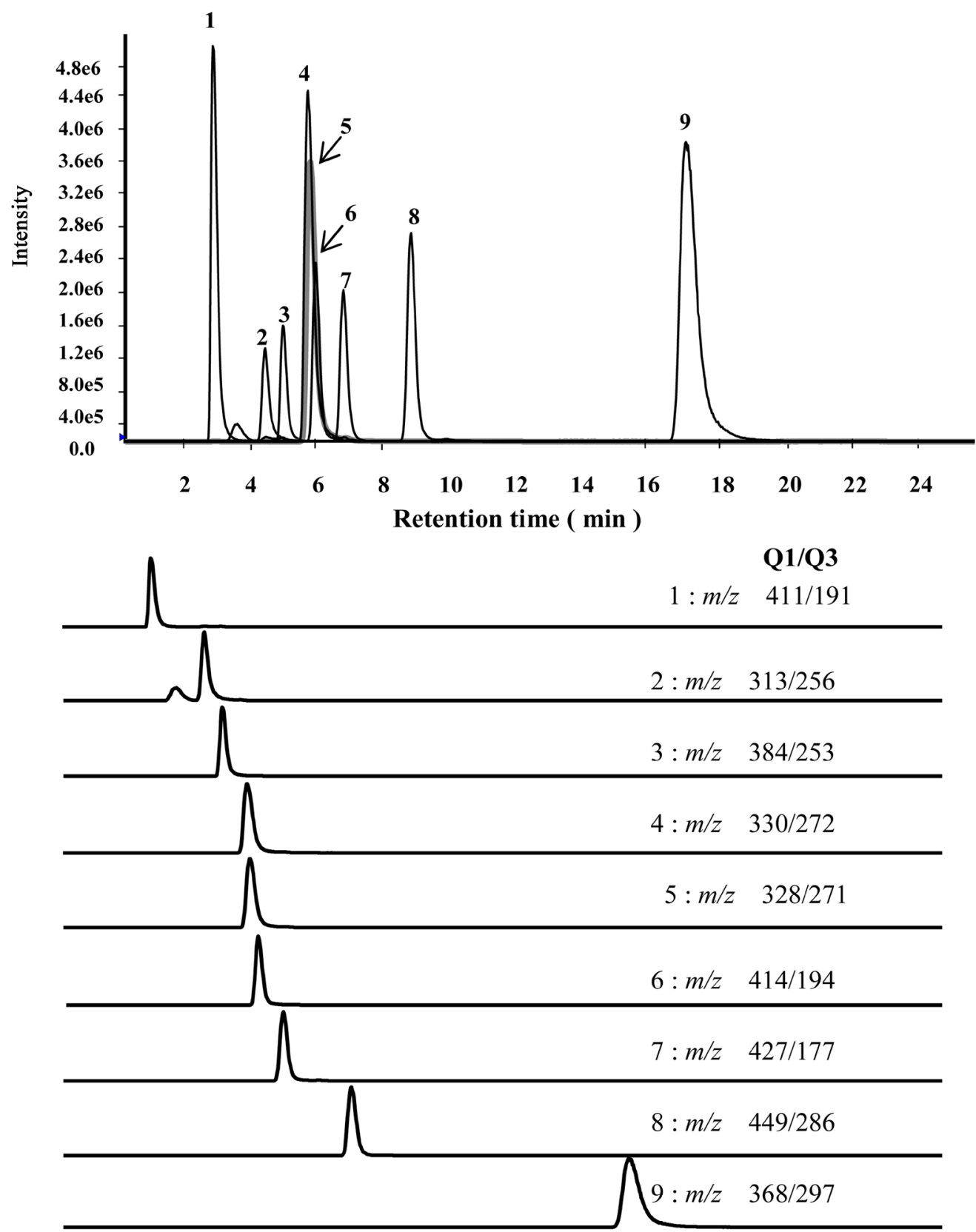

Figure 1. Chromatograms of atypical antipsychotic drugs (50 ng/mL). Column: Mightysil-RP-18 MS column $(2.0 \mathrm{~mm} \times 150 \mathrm{~mm}$, particle size $5 \mu \mathrm{m})$; 1: risperidone, 2: olanzapine, 3: quetiapine, 4: clozapine- $d_{\phi}$ 5: clozapine, 6: ziprasidone, 7: perospirone, 8: aripiprazole, 9: blonanserin.

were stored refrigerated at $4^{\circ} \mathrm{C}$ for same-day analysis, whereas serum samples were frozen at $-20^{\circ} \mathrm{C}$ until they were analyzed by LC/MS/MS.

\subsection{LOD and LOQ}

The LODs of risperidone, olanzapine, quetiapine, clozapine, ziprasidone, perospirone, aripiprazole and blonanserin were $0.00071,0.031,0.015,0.046,0.017$, $0.0057,0.012$ and $0.027 \mathrm{ng} / \mathrm{mL}$, respectively and the LOQs of the eight drugs were $20.0 \mathrm{ng} / \mathrm{mL}$ (Table 3 ). 

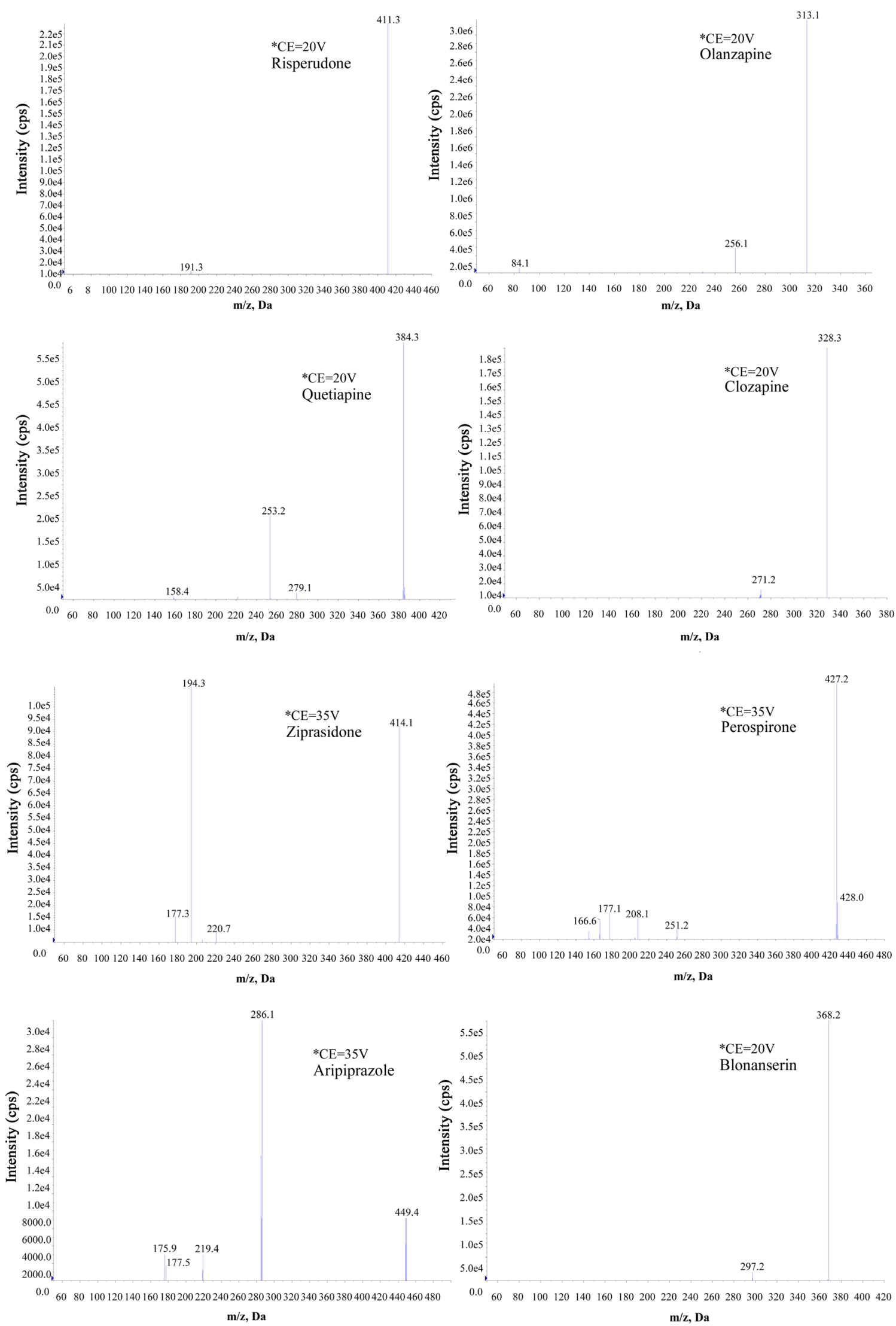

Figure 2. Mass spectra of atypical antipsychotic drugs obtained with LC/MS/MS. ${ }^{\star} \mathrm{CE}$ : Collision Energy. 
Table 2. Calibration curves of eight atypical antipsychotic drugs.

\begin{tabular}{cccc}
\hline Drug & Range $(\mathrm{ng} / \mathrm{mL})$ & \multicolumn{1}{c}{ Curve } & $\mathrm{r}$ \\
\hline Risperidone & $20.0-100.0$ & $\mathrm{y}=0.0135 \mathrm{x}+0.139$ & 0.9936 \\
Olanzapine & $20.0-100.0$ & $\mathrm{y}=0.0696 \mathrm{x}-0.00557$ & 0.9968 \\
Quetiapine & $20.0-100.0$ & $\mathrm{y}=0.0445 \mathrm{x}+0.0165$ & 0.9986 \\
Clozapine & $20.0-100.0$ & $\mathrm{y}=0.012 \mathrm{x}-0.0849$ & 0.9941 \\
Ziprasidone & $20.0-100.0$ & $\mathrm{y}=0.00752 \mathrm{x}-0.0000295$ & 0.9950 \\
Perospirone & $20.0-100.0$ & $\mathrm{y}=0.0056 \mathrm{x}+0.0512$ & 0.9977 \\
Aripiprazole & $20.0-100.0$ & $\mathrm{y}=0.00301 \mathrm{x}-0.0932$ & 0.9177 \\
Blonanserin & $20.0-100.0$ & $\mathrm{y}=0.0122 \mathrm{x}-0.0854$ & 0.9794 \\
\hline
\end{tabular}

Table 3. Recoveries, precision, limit of detection (LOD) and quantification (LOQ) of eight atypical antipsychotic drugs in human serum by LC/MS/MS.

\begin{tabular}{ccccccc}
\hline \multirow{2}{*}{ Drugs } & \multicolumn{3}{c}{ Recovery $(\mathrm{n}=6)$} & \multicolumn{2}{c}{ LOD } & LOQ \\
\cline { 2 - 4 } & $\begin{array}{c}\text { Amount } \\
\text { added }(\mathrm{ng})\end{array}$ & $\begin{array}{c}\text { Average } \\
(\%)\end{array}$ & SD & & $(\mathrm{ng} / \mathrm{mL})$ & $(\mathrm{ng} / \mathrm{mL})$ \\
\hline Risperidone & 50.0 & 97.0 & 0.086 & 0.00071 & 20 \\
Olanzapine & 50.0 & 83.7 & 0.055 & 0.031 & 20 \\
Quetiapine & 50.0 & 94.6 & 0.024 & 0.015 & 20 \\
Clozapine & 50.0 & 95.0 & 0.060 & 0.046 & 20 \\
Ziprasidone & 50.0 & 81.3 & 0.028 & 0.017 & 20 \\
Perospirone & 50.0 & 106 & 0.029 & 0.0057 & 20 \\
Aripiprazole & 50.0 & 140 & 0.044 & 0.012 & 20 \\
Blonanserin & 50.0 & 103 & 0.042 & 0.027 & 20 \\
\hline
\end{tabular}

These results suggest that the detection sensitivity of this method is acceptable for the determination of therapeutic concentrations of these drugs (for example, therapeutic concentration of risperidone is $0.002-0.02 \mu \mathrm{g} / \mathrm{mL}$ ).

Fisher D.S. et al. [8] developed a method for the determination of some antipsychotic drugs (olanzapine, quetiapine, clozapine and aripiprazole) in human plasma and serum by LC/MS/MS. Koo T. S. et al. [10] developed a method for the determination of atypical antipsychotic drug in rat plasma by LC/MS/MS. The LOQ was approximately $2.0 \mathrm{ng} / \mathrm{mL}$ for lurasidone. Nirogi R. V. et al. [11] developed a method for the determination of atypical antipsychotic drug in human plasma by LC/MS/MS with ESI. The LOQ was approximately $0.1 \mathrm{ng} / \mathrm{mL}$ for olanzapine. Our LC/MS/MS with ESI method was developed for simultaneous determination of eight atypical antipsychotic drugs. The method was able to measure compounds at approximately the same concentrations as the analytical methods of Koo T. S. et al., Nirogi R. V. et al. and Fisher D. S. et al. [8] [10] [11]. In our experiments, the LOQs in serum were $20 \mathrm{ng} / \mathrm{mL}$ for risperidone, olanzapine, quetiapine, clozapine, ziprasidone, perospirone, aripiprazole and blonanserin. 


\section{Conclusions}

In gas chromatography-mass spectrometry (GC/MS) analysis, it is necessary to gasify the sample and the analyzable samples are limited to relatively low molecular gas, volatile compound or thermally stable compound. In LC/MS/MS, it is possible to analyze difficult to volatile compounds and thermally unstable compounds as long as they are dissolved in the mobile phase, which has the advantage that the application range of analytical samples is wider than GC/MS [12].

We developed a sensitive and specific LC/MS/MS method for the simultaneous detection and quantification of eight atypical antipsychotic drugs (risperidone, olanzapine, quetiapine, clozapine, ziprasidone, perospirone, aripiprazole and blonanserin) in human serum. Samples were treated prior to the analysis, by solid-phase extraction with an Oasis HLB cartridge column. By using this method, samples were separated with sufficient sensitivity, precision and accuracy and will be useful for the determination of the blood levels of atypical antipsychotic drugs in clinical and forensic investigations.

\section{References}

[1] Grundmann, M., Kacirova, I. and Urinovska, R. (2014) Therapeutic Drug Monitoring of Atypical Antipsychotic Drugs. Acta Pharmaceutica, 64, 387-401. https://doi.org/10.2478/acph-2014-0036

[2] Shastry, C.S., Shafeeque, A.A. and Ashwathnarayana, B.J. (2013) Effect Combination of Aripiprazole with Carbamazepine and Fluvoxamine on Liver Functions in Experimental Animals. Indian Journal of Pharmacology, 45, 121-125. https://doi.org/10.4103/0253-7613.108280

[3] Urichuk, L., Prior, T.I., Dursun, S. and Baker, G. (2008) Metabolism of Atypical Antipsychotics: Involvement of Cytochrome p450 Enzymes and Relevance for Drug-Drug Interactions. Current Drug Metabolism, 9, 410-418. https://doi.org/10.2174/138920008784746373

[4] Ballesteros, S., Martinrz, M.A., Ballesteros, M.A., de la Torre, C.S. and RodriguezBorregan, J.C. (2007) A Case of Olanzapine Overdose with Analytical Data. Clinical Toxicology, 45, 412-415. https://doi.org/10.1080/15563650601072183

[5] Griffiths, C. and Flanagan, R.J. (2005) Fatal Poisoning with Atypical Antipsychotic Drugs, England and Wales 199s3-2002. Journal of Psychopharmacology, 19, 667-674. https://doi.org/10.1177/0269881105056658

[6] Saracino, M.A., Mercolini, L., Flotta, G., Albers, L.J., Merli, R. and Raggi, M.A. (2006) Simultaneous Determination of Fluvoxamine Isomers and Quetiapine in Human Plasma by Mean of High-Performance Liquid Chromatography. Journal of Chromatography B, 843, 227-233. https://doi.org/10.1016/j.jchromb.2006.06.001

[7] Xiong, X., Yang, L. and Duan, J. (2013) Development and Validation of a Sensitive and Robust LC-MS/MS with Electrospray Ionization Method for Simultaneous Quantitation of Quetiapine and Its Active Metabolite Norquetiapine in Human Plasma. Clinica Chimica Acta, 423, 69-74. https://doi.org/10.1016/j.cca.2013.04.016

[8] Fisher, D.S., Partridge, S.J., Handley, S.A., Couchman, L., Morgan, P.E. and Flanagan, R.J. (2013) LC-MS/MS of Some Atypical Antipsychotics in Human Plasma, Serum, Oral Fluid and Haemolysed Whole Blood. Forensic Science International, 229, 145-150. https://doi.org/10.1016/j.forsciint.2013.02.010 
[9] Wen, Y.G., Ni, X.J., Zhang, M., Liu, X. and Shang, D.W. (2012) Simultaneous Determination of Blonanserin and Its Metabolite in Human Plasma and Urine by Liquid Chromatography-Tandem Mass Spectrometry: Application to a Pharmacokinetic Study. Journal of Chromatography B, 903, 46-52. https://doi.org/10.1016/j.jchromb.2012.06.037

[10] Koo, T.S., Kim, S.J., Lee, J., Ha, D.J., Baek, M. and Moon, H. (2011) Quantification of Lurasidone, an Atypical Antipsychotic Drug, in Rat Plasma with High-Performance Liquid Chromatography with Tandem Mass Spectrometry. Biomedical Chromatography: BMC, 12, 1389-1394. https://doi.org/10.1002/bmc.1625

[11] Nirogi, R.V., Kandikere, V.N., Shukla, M., Mudigonda, K., Maurya, S., Boosi, R. and Yerramilli, A. (2006) Development and Validation of Sensitive Liquid Chromatography/Electrospray Tandem Mass Spectrometry Assay for the Quantification of Olanzapine in Human Plasma. Journal of Pharmaceutical and Biomedical Analysis, 41, 935-942. https://doi.org/10.1016/j.jpba.2006.01.040

[12] Giulio, M., Flaminia, P., Roberta, T., Miriam, C., Federica, U.R. and Francesco, P.B. (2015) Development and Validation of a GC-MS METHOD for the Detection and Quantification of Clotiapine in Blood and Urine Specimens and Application to a Postmortem Case. International Journal of Analytical Chemistry, 2015, 1155-1160. 